\title{
Pharmacists Knowledge and contribution during COVID-19 pandemic in Sudan, 2020
}

\author{
Nahid Ahmed ${ }^{1}$ and Ali Saeed ${ }^{1}$ \\ ${ }^{1}$ Napata College
}

July 20, 2020

\begin{abstract}
Introduction: Pharmacist's knowledge about different aspects of this pandemic is crucial because it influence their role and contribution as a frontline health care provider, as pharmacies and most of the pharmacy practice sectors are kept open even during lockdowns providing counseling, patient care. Pharmacist can provide valuable services during COVID-19 pandemic, these services may include: provide reliable information on the disease, participate in public education on preventive measures, referring of suspected cases, insuring continuous supply of medicine. Methods: A web-based, cross-sectional study, conducted using survey instrument to obtain responses from Sudanese pharmacists during the period from 26th of May to 3rd of June 2020. A 14-item survey instrument was developed. The web-based cross-sectional study was carried out among Sudanese pharmacists. A self-reported structured questionnaire was divided into three sections: demographic characteristics, questions assessing the knowledge, and one question for the pharmacist contribution during the pandemic. Results: The study showed that $51.1 \%$ of pharmacists have good knowledge about the COVID-19. The work experience and education level significantly $(\mathrm{P}<0.05)$ influence pharmacist knowledge. Majority of pharmacists contribute to different activities during the pandemic, e.g. providing patients with transmission information (94\%), Provide factual and reliable information on the diseases symptoms (93.1\%), providing patients with prevention information (91.1\%). Conclusion: The study identified that pharmacists have good knowledge about COVID-19 pandemic Also pharmacists contributed in many activities as a frontline health care provider during this pandemic.
\end{abstract}

\section{Introduction:}

The outbreak of Novel corona virus disease 2019 (COVID-19) is the recent worldwide disaster which is considered by the WHO as a Public Health Emergency of International Concern (PHEIC) ${ }^{(1)}$. The first case was discovered in Wuhan City, China ${ }^{(2,3)}$ and then after few weeks spread as a global pandemic ${ }^{(4,5)}$.

In Sudan the first reported COVID-19 positive case was in 11 of March 2020, at the time of writing this article (6 March 2020) there were 9081 reported cases.

The novel corona virus can live on hands, surfaces, objects, mucus membranes, body fluids, respiratory secretions, and spread from person to person through the direct contact ${ }^{(6,7)}$, which makes the most effective preventive measures are avoiding close contact with people, hand hygiene, clean and disinfect surfaces ${ }^{(8)}$, as there is no specific efficacious treatment or vaccine available for this virus until writing of this paper ${ }^{(9,10)}$.

Pharmacist's knowledge about different aspects of this pandemic is crucial because it influence their role and contribution as a frontline health care provider, as pharmacies and most of the pharmacy practice sectors are kept open even during lockdowns providing counseling, patient care ${ }^{(11,12,13)}$.

To strengthen pharmacists knowledge about COVID-19, there are many training programs held worldwide, for example the International Pharmaceutical Federation (FIP), and American Pharmacist Association $(\mathrm{APhA})$ have issued guidance, and resource documents ${ }^{(14,15)}$ 
Although the role of pharmacist especially in the community is ranging between essential and expanded role according to the policies in different countries ${ }^{(16-18)}$, but still pharmacist can provide valuable services during COVID-19 pandemic, these services may include: provide reliable information on the disease, participate in public education on preventive measures, referring of suspected cases, insuring continuous supply of medicine $(19,20)$

\section{Methods}

\section{Survey Instrument and Dissemination}

A web-based, cross-sectional study was conducted using survey instrument to obtain responses from Sudanese pharmacists during the period from $26^{\text {th }}$ of May to $3^{\text {rd }}$ of June 2020.

A 14-item survey instrument was developed. The web-based cross-sectional study was carried out among Sudanese pharmacists. A self-reported structured questionnaire was divided into three sections: demographic characteristics (4 questions), questions assessing the knowledge (9 questions), and one question for the pharmacist contribution during the pandemic. The questionnaire prepared using the Google forms and the link was shared through social media; Facebook, e-mail, and Whatsapp groups. The purpose of the study was explained to participants prior to attending the survey. The participation of the study was voluntary and only those who are willing participated in the survey.

\section{Data Analysis}

For analysis of data, Statistical Package for Social Sciences software, version 21.0 (IBM SPSSInc., Chicago, IL) and STATA 11 were used. Initially, all information gathered via questionnaire then coded into variables. Normality of data was tested using Kolmogorov-Smirnov test and Shapiro-Wilk test. Both descriptive and inferential statistics involving Pearson Chi-Square, Fisher's exact test, Pearson Correlation, One Way ANOVA (Analysis of variances) Test, were used to present results. A p-value of less than 0.05 was considered statistically significant.

Score: In order to have a better assessment of overall knowledge, each correct answer in the knowledge domain carried 1 mark while wrong answer carried 0 mark, also question consist of more than one correct answers one mark was distributed according to number of answers, this gave a total score range of $0-10$ then converted to percentages.

\section{Ethical Considerations}

Survey questions do not include name or contact information, and participation in the study was voluntary.

\section{Results:}

\section{Demographic data:}

Three hundred forty eight pharmacists participated in the study, $62.1 \%$ were female while $37.9 \%$ were male. $57.8 \%$ from the participants were master holders, $38.8 \%$ were bachelor holders and $3.4 \%$ were PhD holder.

Regarding participants' work place, $25.9 \%$ working as community pharmacists, $17.2 \%$ working in pharmaceutical companies and $13.8 \%$ working in hospital pharmacy. (Table 1)

The participants' working experience ranges between more than 10 years' experience $(37.4 \%), 5$ - 10 years' experience (33.9\%), and less than 5 years' experience $(28.7 \%)$.

\section{Table 1: Demographic data}

\begin{tabular}{ll}
\hline Demographic variables & Percent \\
\hline Place of work $(\mathbf{n}=\mathbf{3 4 8})$ & \\
Hospital pharmacy, Community Pharmacy and Company & $0.3 \%$ \\
Clinical pharmacy, Community Pharmacy and Ministry of health & $0.3 \%$
\end{tabular}




\begin{tabular}{ll}
\hline Demographic variables & Percent \\
\hline Community Pharmacy, Academic and Company & $0.3 \%$ \\
Hospital pharmacy and Company & $0.3 \%$ \\
Hospital pharmacy and Academic & $0.6 \%$ \\
Hospital pharmacy and Clinical pharmacy & $0.6 \%$ \\
Community Pharmacy and Company & $0.9 \%$ \\
Clinical pharmacy and Academic & $0.9 \%$ \\
Clinical pharmacy and Ministry of health & $1.1 \%$ \\
Community Pharmacy and Academic & $1.1 \%$ \\
Clinical pharmacy and Community Pharmacy & $1.7 \%$ \\
National medical supply & $2.6 \%$ \\
Hospital pharmacy and Community Pharmacy & $3.2 \%$ \\
Ministry of health & $7.8 \%$ \\
Academic & $9.8 \%$ \\
Clinical pharmacy & $11.8 \%$ \\
Hospital pharmacy & $13.8 \%$ \\
Company & $17.2 \%$ \\
Community Pharmacy & $25.9 \%$ \\
\hline
\end{tabular}

\section{Knowledge of the participants about COVID- 19:}

The majority of participants $(80.2 \%)$ respond with the right answer about the incubation period for the COVID-19 virus which is 2- 14 days.

About the main methods of transmission of COVID-19 virus, $26.4 \%$ and $7.5 \%$ of participants give wrong response about possibility of transmission through fecal- oral route, and raw food respectively. While $82.2 \%$, $82.5 \%, 89.1 \%, 64.4 \%$ and $26.4 \%$ choose the right methods of transmission: close contact from person to person, contaminated surface, coughing and sneezing, air droplets and through body fluids respectively.

About the main signs and symptoms associated with COVID-19, 96.6\%, 95.1\%, 94.8\%, 91.4\% 91.1\% and $68.1 \%$ of participants think that main signs and symptoms are fever, shortness of breath, dry cough, headache, general weakness and diarrhea respectively. Also 27.3\%, 23.3\%, 19\% and 5.2\% think that the symptoms are anorexia, CT ground- glass opacification, insomnia and ageusia respectively. While $2.3 \%$ select the wrong answer edema.

Regarding sever illness risk factors, participants think that chronic illness, older adults, cancer patients, smoker, pregnant women and adult male are at high risk for severe COVID-19, while $7.8 \%$ and $4.6 \%$ select the wrong answers : children and adult female respectively.

Few participants (9.5\%) think that COVID-19 can only gain inside the body through the nose but not through the eyes and mouth. Also only $6 \%$ think that healthy carriers of virus who has no cough or fever cannot spread COVID-19. The majority (98.9\%) know that the best way to prevent COVID-19 is to avoid crowed and stay at home.

As a response to the question about management of mild cases, $73 \%$, and $96.8 \%$ of participants select vitamin $\mathrm{D}$ and vitamin $\mathrm{C}$ respectively, while some of them select wrong answers e.g. Azithromycin (67.5\%), Oxygen (24.4\%). For moderate cases $12.6 \%$ select Amoxicillin, $88.5 \%$ select Azithromycin, and some of them select wrong answers e.g. Hydoxychloroquine (75.3\%), corticosteroids (8.9\%). (Table 2)

As general after responding to the knowledge questions, about half of the participants (51.1\%) in the study have good knowledge about COVID-19.

Table 2: Pharmacists' Knowledge about COVID-19 Pandemic 


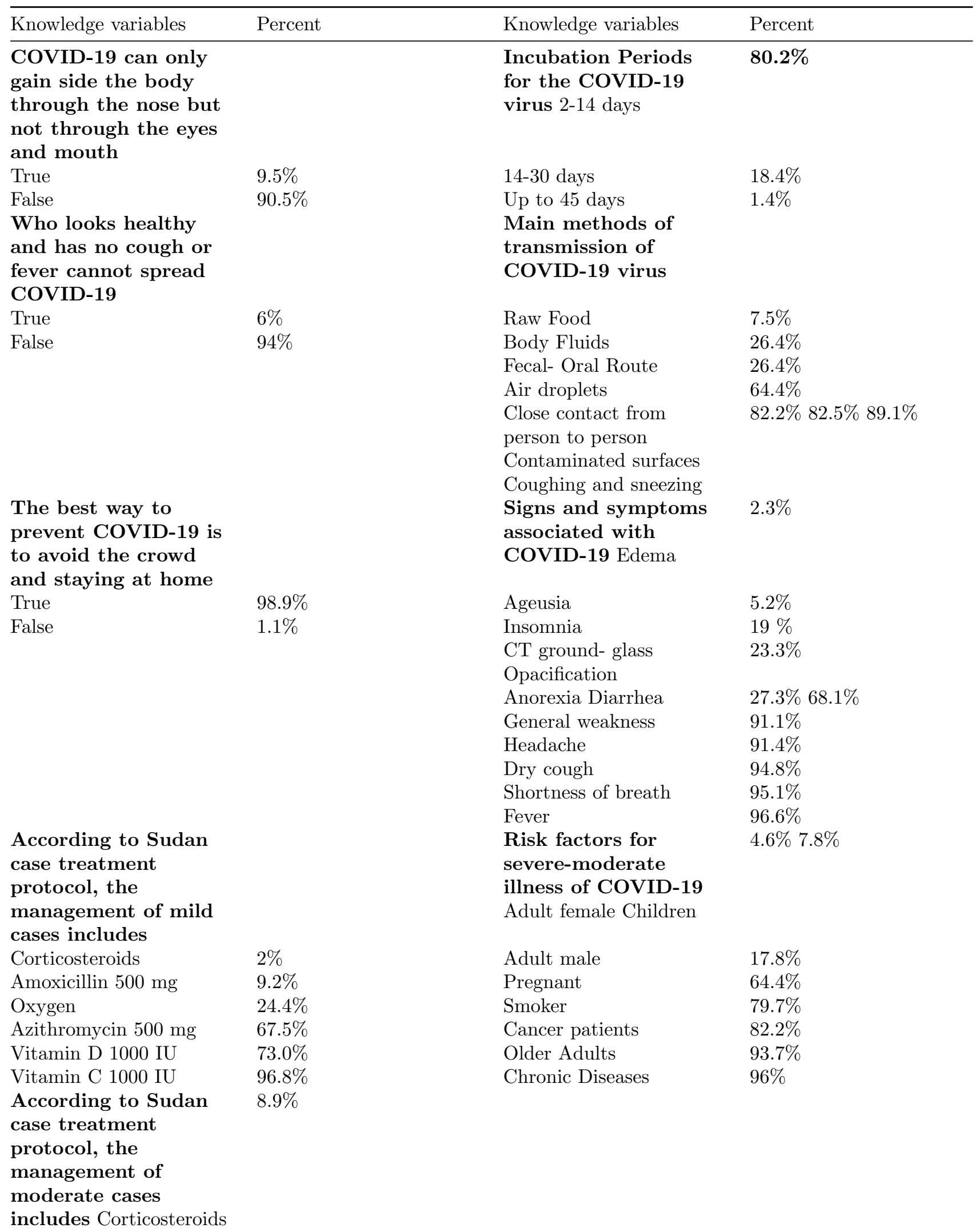




\begin{tabular}{llll}
\hline Knowledge variables & Percent & Knowledge variables & Percent \\
\hline Amoxicillin 500 mg & $12.6 \%$ & & \\
Oxygen & $55.7 \%$ & & \\
Hydroxy chloroquine & $75.3 \%$ & & \\
400mg & & & \\
Azithromycin 500 mg & $88.5 \%$ & \\
\hline
\end{tabular}

\section{Contribution of pharmacists during COVID-19:}

During these pandemic pharmacists as a health care provider participated in different activities. The majority $(94 \%, 93.1 \%)$ provide factual and reliable information on the diseases symptoms and transmission information respectively. And other activities e.g. providing patients with prevention information (Table 3)

Table 3: Pharmacists' contribution during COVID-19 pandemic

\begin{tabular}{ll}
\hline Contribution variables & Percent of yes \\
\hline Participate in developing COVID-19 protocols & $51.7 \%$ \\
Participate in COVID-19 research efforts & $52.9 \%$ \\
Participate in preparation of stock of medications used in of COVID-19 treatment protocols & $59.5 \%$ \\
Referral of suspected patients & $69 \%$ \\
Participate in synthesizing or distributing sanitizers initiatives & $75 \%$ \\
Participate in community awareness programs & $76.7 \%$ \\
Providing patients with prevention information & $91.1 \%$ \\
Provide factual and reliable information on the diseases symptoms & $93.1 \%$ \\
Providing patients with transmission information & $94 \%$ \\
\hline
\end{tabular}

There is a significant relationship between work experience and the participants knowledge about COVID-19 (P-value: 0.043) using Pearson Chi-Square and Fisher's Exact test as shown in table 4.

Table 4: Correlation between variables and the knowledge using Chi-Square Test

\begin{tabular}{lllll}
\hline Variables & Variables & Knowledge score & Knowledge score & Pearson Chi-Square Test P value \\
\hline \multirow{3}{*}{ Gender } & & Poor & Good & \\
& Male & $50.00 \%$ & $50.00 \%$ & $0.737^{*}$ \\
Level of education & Female & $48.10 \%$ & $51.90 \%$ & \\
& Bachelor & $54.10 \%$ & $45.90 \%$ & $0.205^{*}$ \\
& Master & $46.30 \%$ & $53.70 \%$ & \\
Work experience & & $33.30 \%$ & $66.70 \%$ & $0.043^{* *}$ \\
& Less than 5 years & $59.00 \%$ & $41.00 \%$ & \\
& & $42.40 \%$ & $57.60 \%$ & \\
& & $46.90 \%$ & $53.10 \%$ & \\
\hline
\end{tabular}

There is a significant relationship between level of education and knowledge score using one way ANOVATest (P- value 0.035$)$ as shown in table 5.

Table 5: Correlation between variables and the knowledge using one way ANOVA- Test 


\begin{tabular}{lllll}
\hline One Way ANOVA-Test & One Way ANOVA-Test & One Way ANOVA-Test & One Way ANOVA-Test & One Way ANOVA- \\
\hline Variables & Variables & Number & Knowledge score $(\%)$ & $\begin{array}{l}\text { Knowledge score }(\% \\
\text { Std. Deviation }\end{array}$ \\
& & & Mean & 10 \\
Level of education & Bachelor & 135 & 73 & 8 \\
& Master & 201 & 75 & 8 \\
Work experience & PhD & 12 & 78 & 9 \\
& Less than 5 years & 100 & 73 & 9 \\
& 5-10 years & 118 & 75 & 9 \\
\hline
\end{tabular}

\section{Discussion:}

This study revealed that more than half of the pharmacists (51.1\%) have good knowledge about COVID-19 pandemic especially concerning methods of transmission, incubation period, prevention methods and risk of severe illness.

And this type of knowledge is very important as suspected patients and co-patients may reach pharmacists in community pharmacy asking for over the counter medications to relief their symptoms before they seek medical attention, when they get worse, so it is so important that the pharmacists must have the knowledge so as to council those patients, more over this good know edge may help pharmacists to protect themselves from getting infected.

Nevertheless their knowledge about treatment guidelines should be better, for example more than $67 \%$ of participants, and more than $24 \%$ think that Azithromycin and oxygen respectively is used in the national protocol for treatment of mild cases, while in fact they are not.

Also more than $75 \%$ of pharmacists think that hydroxychloroquine is used for treatment moderate cases, while according to the Sudan national protocol it is used for the treatment of severe cases.

Although the Sudan national treatment protocol for COVID-19 is available in the Federal Ministry of Health website $^{(21)}$ but it is not widely distributed in the media so pharmacists do not have the sufficient knowledge about this protocol.

Regarding contribution, the majority of participants provide different types of information e.g: information about diseases symptoms (93.1\%), transmission information (94\%), and prevention information (91.1\%).

Different initiatives were acting in Sudan during this pandemic; pharmacists play many roles in these initiatives e.g.: synthesizing or distributing sanitizers (75\%), also $76.7 \%$ of pharmacists participate in community awareness programs.

More than half of pharmacists participate in referral of suspected patients, developing protocols, research efforts, preparing medication stocks.

The study revealed that work experience and level of education significantly $(\mathrm{P}<0.05)$ influence pharmacists knowledge about the COVID-19 pandemic.

\section{Limitations}

This study has the limitations of online cross sectional studies which depends on participants' honesty, and the limited sample size, which may limit the generalization of the results.

\section{Conclusion}

We identified that pharmacists have good knowledge in different aspects regarding COVID-19 pandemic, we found that the level of education and work experience influence this knowledge.

Also pharmacists contributed in many activities as a frontline health care provider during this pandemic. 


\section{References:}

1. Euro surveillance Editorial Team, Note from the editors: World Health Organization declares novel coronavirus (2019-nCoV) sixth public health emergency of international concern, Euro Surveill, 2020 Feb;25 (5): pii= 200131e, https: //doi.org/10.2807/1560-7917. ES.2020.25.5.200131e.

2. Hengbo Zhu, Li Wei and Ping Niu, The novel coronavirus outbreak in Wuhan, China, Global Health Research and Policy, 2020, 5:6, 1-3.

3. Jean-claude Perez, Wuhan Covid-19 Synthetic Origins and Evolution, International Journal of Research - Granthaalayah 8(2), 285-324, (February 2020):285-324.

4. Abdulazeez Adeyemi Anjorin, The coronavirus disease 2019 (COVID-19) pandemic: A review and an update on cases in Africa, Asian Pacific Journal of Tropical Medicine, April 2020, 13(4):1-5.

5. Coronavirus disease 2019 (COVID-19) pandemic: increased transmission in the EU/EEA and the UK -seventh update, 25 March 2020, European center for disease prevention and control, PP 1-5

6. Meng L, Hua F, Bian Z., Coronavirus disease 2019 (COVID-19): emerging and future challenges for dental and oral medicine, Journal of Dental Research, 2020 March, Vol. 99(5), 481-487.

7. Peng X, Xu X, Li Y, Cheng L, Zhou X, Ren B, Transmission routes of 2019-nCoV and controls in dental practice, International Journal of Oral Science, 2020 March 3; 12 (1):1-6.

8. How to protect yourself and others, Centers of Disease Control and Prevention Coronavirus disease (COVID-2-19), available on https://www.cdc.gov/ coronavirus/ 2019-ncov/prevent- gettingsick/prevention.html (Accessed May 2020).

9. J.H. Beigel, , K.M. Tomashek, L.E. Dodd et al , Remdesivir for the Treatment of Covid-19 - Preliminary Report, The new England journal of medicine, May 22, 2020, PP 1-12.

10. Centers for Disease Control and Prevention, Information for Clinicians on Investigational Therapeutics for Patients with COVID-19, Updated April 25, 2020 Available on: https://www.cdc.gov/ coronavirus/2019-ncov/hcp/therapeutic-options.html, (Accessed may 2020).

11. Press release UK Government (April 2, 2020) £300 million announced for community pharmacies to support them during coronavirus outbreak, Available on: https://www.gov.uk/ government/ news/ 300-millionannounced-for-community-pharmacies-to-support-them-during-coronavirusoutbreak. Accessed 05 April 2020.

12. Rutter V, Chan AHY, Tuck C, Bader L, Babar Z-U-D, Bates I, Weaving the health and pharmaceutical care agenda through the themes of the commonwealth heads of government meeting (CHOGM), London 2018, Journal of Pharmaceutical Policy and Practice, 2018; 11 (1):10.

13. Pharmaceutical Group of European Union. Press Release-Community pharmacists in the frontline in the fight against the novel corona-virus disease (COVID-19). Available on: https://www.pgeu.eu/ publications/pressrelease- community-pharmacists-in-the-frontline-in-the-fight against- the novel - corona-virus-disease-covid-19/. Accessed May 2020.

14. International Pharmaceutical Federation. Coronavirus/COVID-19 preparedness. Available on: https: //www.fip.org/coronavirus. Accessed 05 April 2020.

15. American Pharmacist Association. Pharmacists' Guide to Coronavirus. Available on: https://www . pharmacist.com/coronavirus. Accessed 05 April 2020.

16. Cope LC, Abuzour AS, Tully MP, Non-medical prescribing: where are we now? Therapeutic Advances in Drug Safety, 2016; 7 (4):165-172.

17. Hughes CM, Hawwa AF, Scullin C, et al, Provision of pharmaceutical care by community pharmacists: a comparison across Europe, Pharmacy World and Science, 2010; 32 (4):472-487.

18. Aly M, Garcia-Cardenas V, Williams K, Benrimoj SI, A review of international Pharmacy based minor ailment services and proposed service design model, Research in Social and Administrative Pharmacy, 2018; 14(11):989-998.

19. Watson KE, Singleton JA, Tippett V, Nissen LM, Defining pharmacists' roles in disasters: a Delphi study, PloS One, 2019;14 (12):e0227132.

20. Coronavirus SARS- CoV-2 / COVID-19 pandemic: Information and interim guidelines for pharmacists and the pharmacy workforce, 19 March 2020, International Pharmaceutical Federation, FIP Health Advisory (Accessed May 2020), Available from: https://www.fip.org/files/ content/priority-areas/ 
coronavirus/ Coronavirus-guidance-update-ENGLISH.

21. Case treatment protocol for COVID 19 in adults, available on: http://www.sho.gov.sd/controller/ knowledge_hub.php?sm_id=133\&mid=110\&lid=1(Accessed May 2020). 